\title{
MODELAGEM DE PROBLEMAS DE REAÇÃO EM CATALISADORES POROSOS SUJEITOS À LIMITAÇÃO DIFUSIONAL INTERNA DE CALOR E MASSA UTILIZANDO UM AMBIENTE COMPUTACIONAL INTERATIVO DESENVOLVIDO EM MAPLE
}

\author{
Fabiana Vassallo Caldas ${ }^{1}$ \\ Rosenir Rita de Cassia Moreira da Silva \\ Arlindo de Almeida Rocha ${ }^{1}$
}

\begin{abstract}
Resumo: Este trabalho apresenta a aplicação de um software de matemática o "MAPLE" como um meio didático para cálculos complexos especialmente relacionados às reações catalíticas que ocorrem em catalisadores porosos, com diversas geometrias, sujeitos às limitações difusionais internas à transferência de massa e calor, simultaneamente. O problema foi formulado a partir das equações gerais da conservação de massa e energia, utilizando uma rotina desenvolvida para o Software MAPLE, particularizado e solucionado numericamente. Calculou-se o fator de efetividade em função do módulo de Thiele para grãos esféricos, cilíndricos e em forma de placa plana, devido à característica interativa do ambiente desenvolvido. Foram obtidos também os perfis adimensionais de concentração e de temperatura no interior de um grão esférico para reações exotérmicas e endotérmicas.
\end{abstract}

Palavras chaves: MAPLE. Reação catalítica heterogênea. Difusão.

\begin{abstract}
This paper is related to the application of state of the art of MAPLE software in problems related to internal diffusion limitations associated with simultaneous heat and mass transfer in catalytic chemical reactions in porous catalysts with different geometric forms. The problem is formulated starting from the basic general microscopic equations of mass and energy conservation using specific procedures developed for the MAPLE software, resulting in non-linear partial differential equations which are difficult to solve. The problems generated were solved numerically and the solutions are presented in the form of graphics showing the effectiveness factor versus Thiele Modulus. The concentration and temperature profiles inside of the catalytic particles are also presented for exothermic and endothermic reaction.
\end{abstract}

Keywords: MAPLE. Heterogeneous Catalytic Reaction. Diffusion.

\section{INTRODUÇÃO}

A grande maioria dos processos químicos da atualidade são processos catalíticos. Segundo FIGUEIREDO (1989) pode-se afirmar que mais de $80 \%$ dos produtos da indústria química envolvem pelo menos uma etapa catalítica. Nos últimos anos, várias pesquisas têm sido realizadas levando a consideráveis melhorias em processos catalíticos industriais. Neste contexto, a catálise se tornou uma poderosa área de pesquisa acadêmica e industrial. Dentre os catalisadores de maior interesse industrial, destacam-se os catalisadores heterogêneos, geralmente constituídos de sólidos inorgânicos porosos ou não porosos, mássicos ou suportados, de diferentes áreas específicas, contendo sítios ativos que irão efetivamente catalisar a reação.

Diversas etapas consecutivas devem ocorrer durante uma reação catalítica para que esta se torne efetiva, envolvendo transporte de massa dos reagentes e produtos externamente à partícula e no interior dos poros e etapas químicas de adsorção de reagentes, reação e dessorção de produtos (FIGUEIREDO, 1989; FOGLER, 2002).

\footnotetext{
${ }^{1}$ Departamento de Engenharia Química e de Petróleo - UFF - rosenir@vm.uff.br
} 
A velocidade global da reação depende da velocidade de cada uma dessas etapas. Se houver limitações difusionais internas ou externas, esperam-se velocidade ou taxas de reações menores. No entanto, se houver também limitações à transferência de calor, as velocidades de reação podem se comportar de forma diferente, principalmente em reações exotérmicas, levando ao aumento considerável dessas taxas provocadas por aumentos consideráveis da temperatura na superfície e no interior das partículas de catalisador, uma vez que a condutividade térmica efetiva desses materiais é normalmente baixa. Neste caso, podem ocorrer situaçôes de múltiplos estados estacionários que correspondem à instabilidade físico-química (WEISZ, 1962; MAYMO, 1966; FROMENT, 1979).

Para modelar tais sistemas, é possível a partir de balanços microscópicos de massa e de energia chegar-se a um sistema de equações diferenciais que considere todas as possibilidades de variações, mas cuja solução é difícil de obter-se analiticamente (CREMASCO, 2004; INCROPERA, 2003).

Atualmente, com o surgimento de diversos softwares matemáticos tais dificuldades podem ser superadas. Em particular, o software computacional MAPLE permite uma abordagem seqüencial do problema com a possibilidade de executar manipulação algébrica, através do sistema de equaçôes resultante, apresentando uma melhor visualização de como cada um dos parâmetros influencia o processo (MEADE, 1996; ABEL,1999).

O software Maple representa um sistema de computação algébrica e simbólica, tendo isso como sua principal característica e seu poderoso diferencial (HECK, 1993; ABEL, 1999). Portanto, o MAPLE permite resolver problemas determinando a forma analítica e exata de soluções sempre que esta existir, assim como permite que se utilizem variáveis simbólicas ao invés de determinar valores na resolução de um problema. $\mathrm{O}$ MAPLE é um software que permite trabalhar com equaçōes algébricas e diferenciais ordinárias e parciais; construir gráficos; também contando com pacotes de soluções numéricas. Outro recurso que merece destaque é o da programação própria, muito simples, porém bastante poderosa que permite a construção de rotinas a fim de determinar a solução matemática do problema a ser analisado. Além disso, esse software pode executar a tradução da linguagem de programação do MAPLE para códigos como $\mathrm{C}$, Fortran ou mesmo linguagem utilizada no software MATLAB.

O MAPLE apresenta certa vantagem sobre os simuladores de processo, pois é possível acompanhar o processo de solução com uma visão crítica, analisando passo a passo o que está sendo calculado, fato este que enfatiza sua aplicação como software educacional. Além desse fato, consiste num otimizador de tarefas, pois dispensa esforços com procedimentos algébricos ou soluções de equações diferenciais durante a resolução de um exercício.

Diante de todas as vantagens apresentadas, o objetivo do presente trabalho é utilizar o MAPLE para modelar o sistema de equações diferenciais resultantes de reações químicas catalíticas sujeitas às limitações difusionais internas à transferência de calor e massa, apresentando uma abordagem seqüencial.

\section{FORMULAÇÃO DO PROBLEMA}

O problema a ser estudado considera a reação química catalítica irreversível $\mathrm{A} \rightarrow \mathrm{B}$, cuja taxa de reação $\left(-r_{A}\right)$ é de primeira ordem em relação à concentração de $\mathrm{A}\left(C_{A}\right)$, ocorrendo num grão de catalisador, sujeito às limitaçōes difusionais internas à transferência de calor e massa.

Os grãos podem ter geometrias esféricas, cilíndricas e de placa plana.

Partindo-se das equações de conservação de massa e de energia (CREMASCO, 2003; INCROPERA, 2004), em regime permanente e considerando-se que os fluxos radiais predominam, obtém-se para um catalisador esférico, por exemplo, as seguintes equações diferenciais:

$$
\begin{aligned}
& \frac{D_{A, \text { ef }}}{r^{2}} \frac{d}{d r}\left(r^{2} \frac{d C_{A}}{d r}\right)-r_{A}=0 \\
& \frac{\lambda}{r^{2}} \frac{d}{d r}\left(r^{2} \frac{d T}{d r}\right)+\left(-\Delta H_{R}\right)\left(r_{A}\right)=0
\end{aligned}
$$

Sendo $\left(-r_{A}\right)=k C_{A}$, onde $k$ é a constante de Arrhenius que depende da temperatura e pode ser descrita como $\quad k=k_{o} \exp (-E / R T)$. Desta forma, verifica-se que a taxa de reação é uma função fortemente dependente da concentração e da temperatura.

As condições de contorno são definidas na superfície e no centro de forma a garantir sime- 
tria radial e podem ser relacionadas a seguir, pelas equações (3) e (4):

$$
\begin{array}{lll}
\frac{d C_{A}}{d r}=0 & \frac{d T}{d r}=0 \quad \text { em } & r=0 \\
C=C_{A s} & T=T_{s} \quad \text { em } & r=R
\end{array}
$$

A solução dessas equações para determinação dos perfis de concentração e de calor ao longo de $r$ é difícil de ser obtida analiticamente, pois devem ser resolvidas simultaneamente. No entanto, é possível, obter-se uma relação analítica entre a concentração e a temperatura em cada ponto do grão, conforme a equação (5).

$$
T-T_{s}=\frac{\left(-\Delta H_{r}\right) D_{A, e f}}{\lambda}\left(C_{A s}-C_{A}\right)
$$

Para resolver este problema é importante definir-se o módulo de Thiele $(\phi)$ e o fator de efetividade ( $\eta)$. O módulo de Thiele é uma medida da razão entre uma velocidade de reação na superfície e uma velocidade de difusão através da partícula de catalisador, sendo expressa através da equação (6) para um catalisador esférico.

$$
\phi=R \sqrt{\frac{k_{s}}{D_{A, e f}}}
$$

sendo: $k_{S}=k_{O} e^{-\frac{E}{R T_{S}}}$

Assim, quando o módulo de Thiele é grande, a difusão interna normalmente limita a velocidade global de reação e, quando é pequeno, a reação normalmente é a etapa limitante. O módulo de Thiele é avaliado na temperatura da superfície externa, assim, a constate da taxa de reação, $\mathrm{k}_{\mathrm{S}}$, é calculado utilizando-se a expressão de Arrhenius nas condições de $T_{s}$.

O fator de efetividade, $\eta$, expressa a razão entre a taxa real, $\left(-r_{A}\right)$, sujeita a limitação difusional e a taxa intrínseca (sem limitação), podendo se expressa pela equação (8).

$$
\eta=\frac{-r_{A}}{\left(-r_{A}\right)_{S}}
$$

Sendo $\left(-r_{A}\right)_{S}$ a taxa de reação sem limitação, isto é, a taxa que resultaria se toda a superfície do interior da partícula fosse exposta às condições da superfície externa, $C_{A S}$ e $T_{S}$.
Os fatores de efetividade para reaçôes com limitação interna à transferência de massa, mas sem limitação à transferência de calor, variam de 0 (zero) a 1 (um). Valores próximos a 1 indicam que as limitações difusionais são desprezíveis, diz-se que há controle cinético, situação que é desejável. No cálculo de $\eta$, pequenas variações ocorrem em função da ordem da reação e da geometria do catalisador. Para reações sujeitas também à limitação a transferência de calor interna, no caso de reações exotérmicas, pode haver um superaquecimento interno dos grãos catalíticos, resultando num aumento local da taxa de reação, por maior geração de calor que não é transferido adequadamente ao meio, fazendo com que os fatores de efetividade sejam maiores do que 1 (um). Dependendo da entalpia de reação, $\left(\Delta \mathrm{H}_{R}\right)$, os valores de $\eta$ podem atingir valores muito grandes para uma faixa intermediária do módulo de Thiele. Pode levar também a regiōes de operação com múltiplos estágios estacionários, para módulos de Thiele menores do que 1, principalmente quando o grão catalítico apresentar baixa condutividade térmica (FOGLER, 2002; SATERFIELD, 1991; FIGUEIREDO, 1989).

Introduzindo se os parâmetros $\gamma$ e $\beta$ e as variáveis adimensionais sugeridas por Weisz e Hicks (1966), definidas pelas equações (9), (10) e (11), chega-se a forma adimensional da equação (1), representada pela equação (12).

$$
\begin{aligned}
\gamma & =\frac{E}{R T_{s}} \\
\beta & =\frac{\left(-\Delta H_{R}\right) D_{A, e f} C_{A s}}{\lambda T_{s}} \\
x & =\frac{r}{R} \quad e \quad y=\frac{C}{C_{A s}}
\end{aligned}
$$

Após as devidas substituições chega-se a seguinte equação para a determinação dos perfis de concentração no interior da partícula catalítica.

$$
\frac{d^{2} y}{d x^{2}}+\frac{2}{x} \frac{d y}{d x}=\phi^{2} y \exp \left(\frac{\gamma \beta(1-y)}{1+\beta(1-y)}\right)
$$

com as seguintes condições de contorno:

$$
\begin{array}{ll}
\text { C.C. } 1: & \frac{d y}{d r}(0)=0 \\
\text { C.C. } 2: & y(1)=1
\end{array}
$$


A resolução dessa equação não é trivial, pois trata-se de um problema não linear de elevada complexidade, inclusive possuindo soluções múltiplas para certos valores dos parâmetros.

A importância relativa das limitações difusionais internas pode então ser determinada calculando-se o fator de efetividade pela equação (8).

Diversos processos industriais apresentam condiçōes reacionais sujeitas às limitações difusionais. Na reforma a vapor do gás natural, com os tamanhos de partículas de catalisadores relativamente grandes usados industrialmente, estes efeitos resultam em fatores de efetividade muito baixos. Dependendo das condiçōes, tem-se verificado, fatores de efetividade para o catalisador da ordem de 0,3 na região de entrada e talvez da ordem de 0,01 na região de saída dos reformadores. Devido a isto, a atividade aparente aumenta com o decréscimo do tamanho do catalisador por diminuir as limitações difusionais, mas em contrapartida um aumento na queda de pressão ao longo do reformador restringe o tamanho do catalisador aos empregados industrialmente (TWIGG, 1980, NIJEMEISLAND, 2004).

Quanto ao parâmetros $\gamma$ e $\beta$, propostos por Weisz e Hicks (1966), valores típicos para processos industriais variam de $\gamma=6,5(\beta=0,025, \phi=0,22)$ para a síntese do cloreto de vinila a partir de $\mathrm{HCl}$ e acetona, a $\gamma=29,4\left(\beta=6,5 \times 10^{-5}, \phi=1,2\right)$ para a síntese da amônia (FOGLER, 2002; FIGUEIREDO, 1989).

O estudo criterioso e a determinação desses parâmetros, bem como do fator de efetividade, permitem a determinação das condiçõos operacionais e do tamanho dos catalisadores que descartem os efeitos difusivos ou, pelo menos, se não forem possíveis de serem eliminados, que possam ser minimizados.

\section{O AMBIENTE COMPUTACIONAL INTERATIVO}

O ambiente computacional desenvolvido possibilita descrever este problema de forma interativa. Pode-se acompanhar todas as etapas do processo, variando-se os diversos parâmetros. A modelagem desse fenômeno facilita o estudo de cinética heterogênea, resolvendo numericamente as equações que governam o problema (CALDAS, $2005 \mathrm{a}, \mathrm{b})$. Para facilitar o manuseio do ambiente computacional interativo, a modelagem abordada foi dividida em etapas, apresentadas na Figura 1, 2, 3 e 4.

O ambiente utilizado é estruturado em módulos, onde por meio de um menu principal o usuário seleciona o módulo desejado e pode acompanhar na seqüência todos os passos necessários para a obtenção da solução de qualquer problema de transmissão de calor, transferência de massa e quantidade de movimento. A Figura 1 apresenta a tela do menu principal, onde cada módulo pode ser acessado apertando o botão de “+” à esquerda do modulo desejado.

Ao acessar o primeiro módulo correspondente às equaçōes da conservação no sistema de coordenadas adequado o usuário deverá entrar com o sistema de coordenadas que será utilizado para solução do problema: cartesiano, cilíndrico ou esférico. A partir daí o software gera automaticamente as equações gerais da conservação de massa (para um componente puro e para misturas binárias), energia e quantidade de movimento. A Figura 2 mostra o resultado final das equações geradas..

Inicialmente, a análise microscópica engloba as equaçôes da conservação de energia e da massa, que são apresentadas em suas formas gerais para diversos sistemas de coordenadas. Em seguida, efetuam-se as simplificações necessárias, definidas pelo próprio usuário, bem como a adimensionalização do problema. Resolve-se o sistema de equaçốes, no presente caso, uma diferencial (equação 12) e outra algébrica (equação 5).

A rotina apresentada $p$ ara resolução do problema pode ser usada tanto para geometria esférica, como para cilíndrica e retangular, utilizando-se as equações pertinentes e considerando-se as hipóteses referidas acima. Além disso, pode-se modelar o comportamento de sistemas reacionais de ordem zero, primeira e segunda ordem. No presente estudo apenas exemplificaram-se casos de reações de primeira ordem.

No módulo SIMPLIFICAÇÕES é realizada a simplificação das equações, para isso o usuário deverá fornecer os seguintes dados:

- componentes do vetor velocidade e a dependência funcional das mesmas e a dependência funcional da pressão e as componentes da aceleração da gravidade;

- dependência funcional da temperatura e a dependência funcional da taxa de geração de calor por unidade de volume; 
- dependência funcional da concentração de componente A e a expressão da taxa de reação de A por unidade de volume.

Após o fornecimento das simplificações, estas são efetivamente aplicadas a cada equação de conservação. Posteriormente, no módulo PROBLEMA FORMULADO são evidenciadas as equaçôes a serem resolvidas para o problema pro- posto e suas respectivas condições de contorno, conforme pode ser observado pela Figura 3.

A definição de parâmetros para a determinação da equação (8) pode ser visualizada na Figura 4.

Além da formulação do problema esse é resolvido numericamente, a sua solução é apresentada na forma de gráficos de maneira a facilitar a compreensão dos dados.

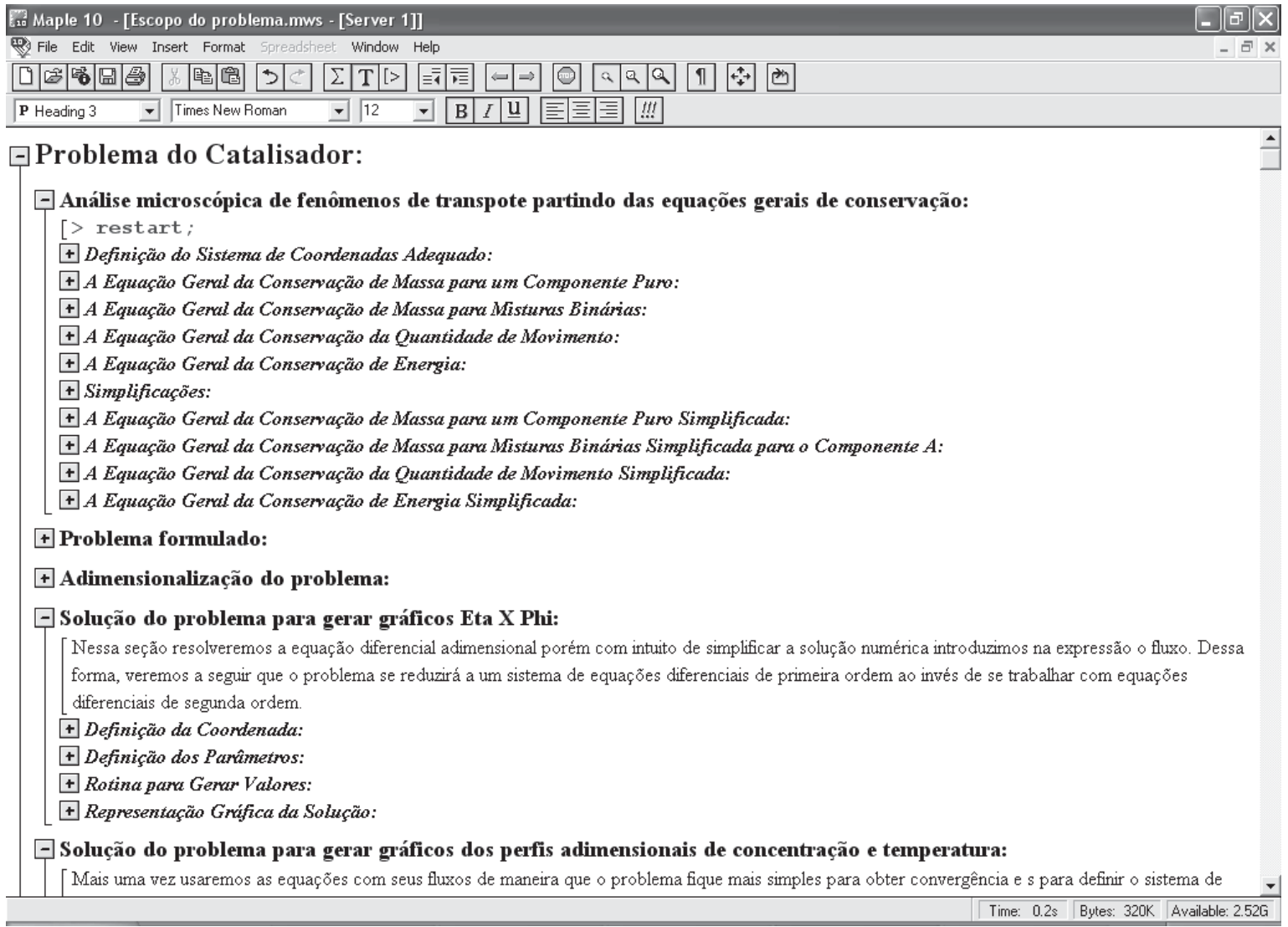

Figura 1. Demonstração do Ambiente Computacional Interativo (CALDAS, 2005a, b)

\section{RESULTADOS OBTIDOS}

Para o problema em questão, foram escolhidos valores de $\gamma$ entre 20 e $40, \beta=-0.3,0,0,1,0,2$ e 0.3 e um intervalo de $\phi$ de 0.2 a 20 , como exemplo. $\mathrm{O}$ valor de $\beta$ negativo corresponde às reações endotérmicas, o positivo para reações exotérmicas e o nulo para reações isotérmicas.

A partir desses valores foram executadas as rotinas construídas resolvendo numericamente a equação obtida. Os gráficos para o fator de efetividade $(\eta)$ versus os valores de $\phi$ para coordenadas esféricas e cilíndricas são apresentados nas Figuras 5 e 6.

Para reações exotérmicas $(\beta>0)$, devido ao aumento significativo da temperatura na superfí- cie e no interior dos catalisadores, as taxas de reação tornam-se maiores resultando em valores altos para o fator de efetividade, maiores do que 1. Para valores elevados de $\beta$ e na zona dos baixos valores de $\phi$, correspondem três valores de $\eta$ não mostrados no intervalo analisado para o exemplo. Este comportamento resulta da solução da equação do balanço de energia ser uma função fortemente não linear da temperatura, conduzindo a soluções múltiplas da equação, o que corresponde a uma instabilidade físico-química. Nessa situação de múltiplo estado estacionário somente os valores mais altos ou mais baixos de $\eta$ são atingidos conforme a direção da aproximação, correspondendo os valores médios a um estado instável. 


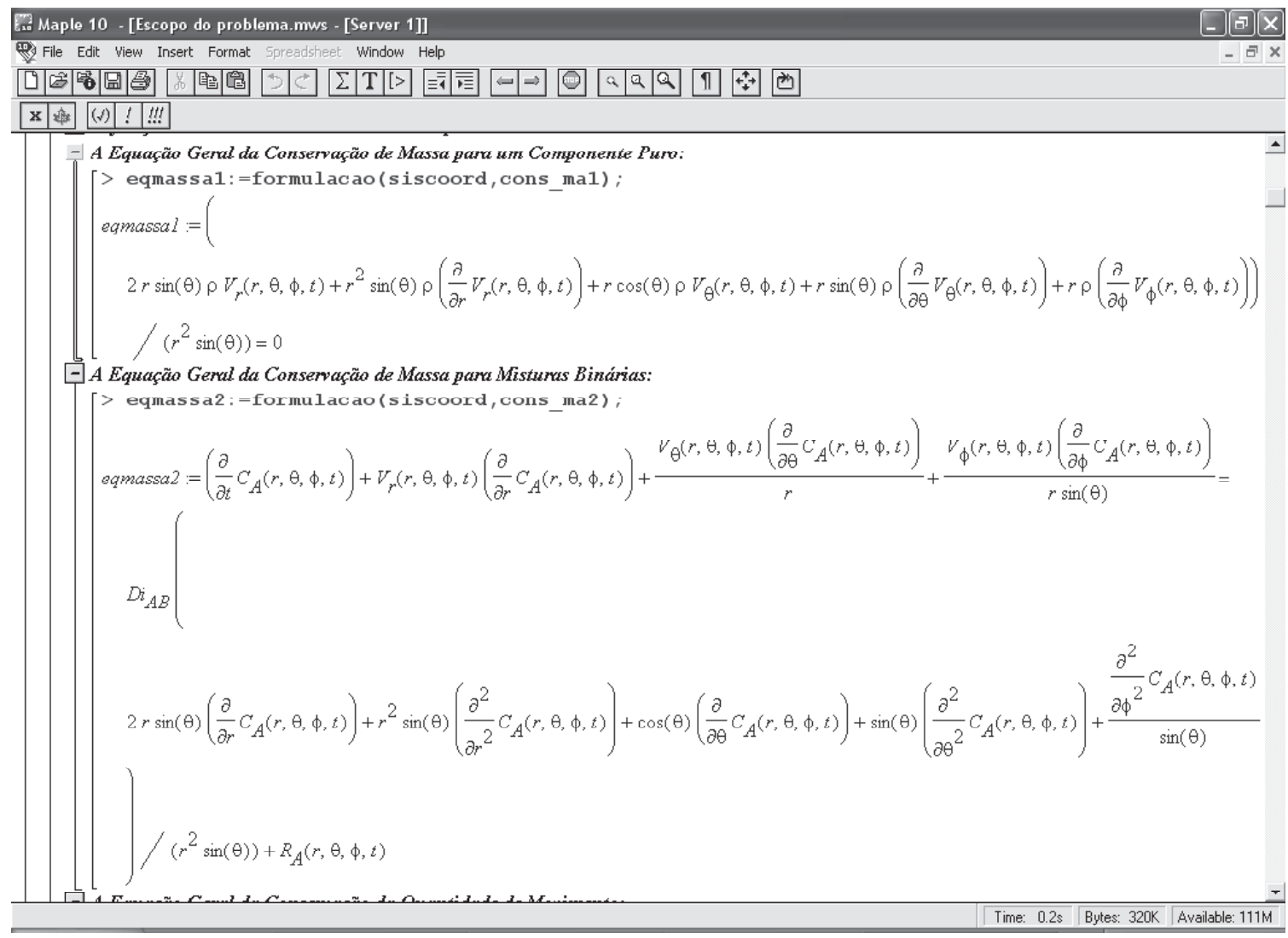

Figura 2. Equações Gerais da Conservação no MAPLE (CALDAS, 2005a, b)

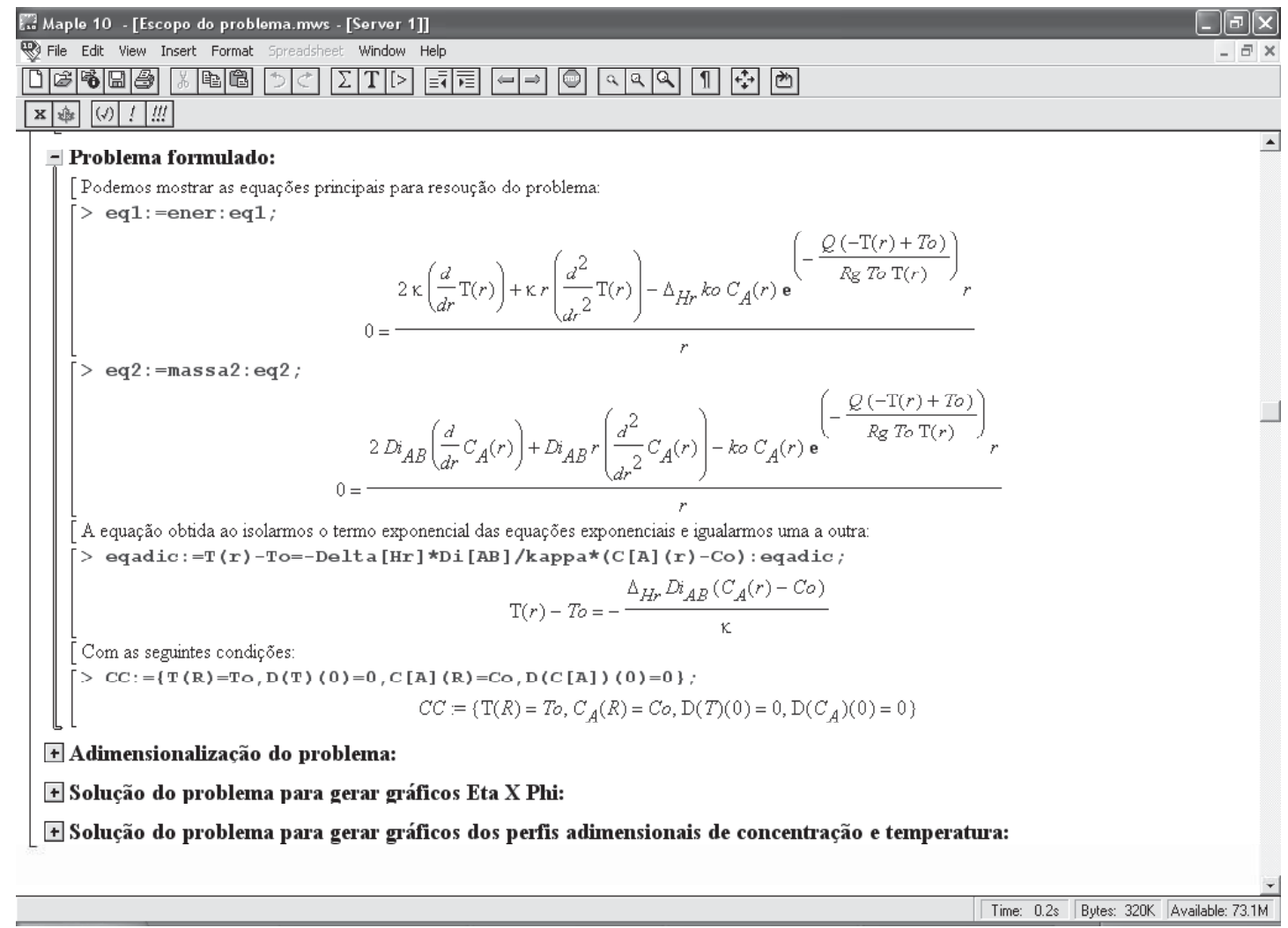

Figura 3. Simplificação das equações de conservação de massa e energia e obtenção da relação analítica entre temperatura e concentração (CALDAS, 2005a, b) 


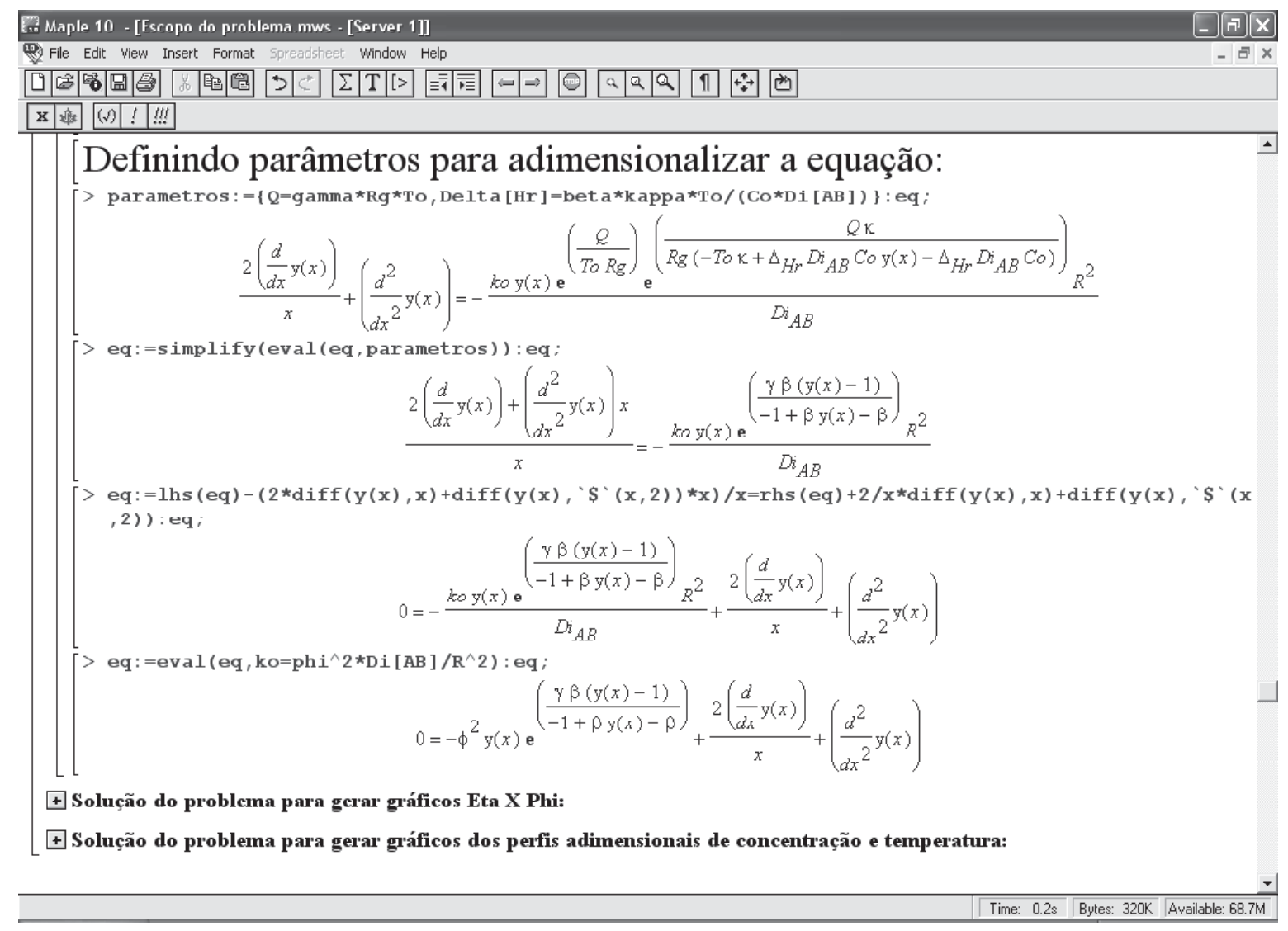

Figura 4. Adimensionalização das equações (CALDAS, 2005a, b)

Pelas Figuras 5 e 6, verificam-se que os fatores de efetividade, para reações catalíticas exotérmicas sujeitas às limitações difusionais, chegam a atingir valores mil vezes maiores do que os obtidos para reações endotérmicas. O grau de exotermicidade da reação se mostrou mais influente do que a geometria do catalisador, sendo os fatores de efetividade observados para os catalisadores esféricos e cilíndricos similares, da mesma ordem de grandeza. Em trabalho anterior, CALDAS et. al. (2005 a, b) comparando resultados com diferentes geometrias, incluindo também os catalisadores na forma de placas planas, também chegou a resultado semelhante.

Nas Figuras 7 e 8, também se verificam os perfis adimensionais de concentração e temperatura em função da distância, em coordenadas esféricas, para reações endotérmicas e exotérmicas, respectivamente. Esses perfis são bem interes- santes, pois permitem visualizar o que realmente ocorre no interior do grão, além de não ser disponibilizado na literatura.

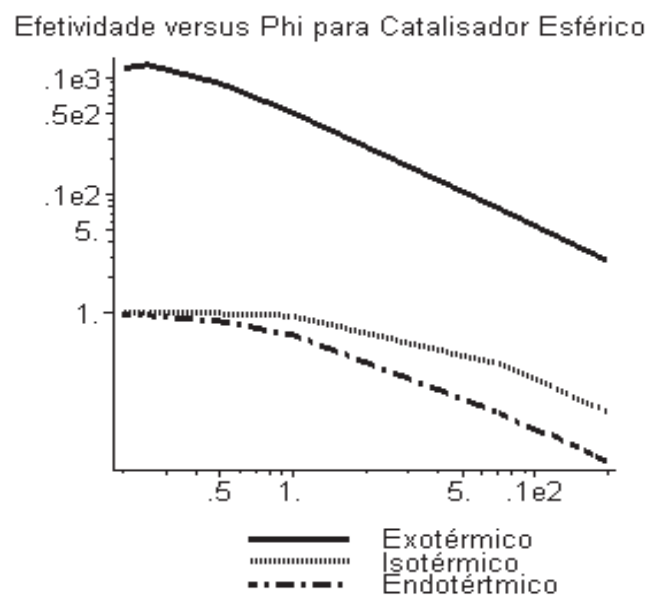

Figura 5. Fator de Efetividade $(\eta)$ em função do Módulo de Thiele $\phi$ (Phi) para catalisador esférico 
Para reações na qual a limitação difusional interna exerce extrema importância, verificase que a reação química ocorre quase completamente na superfície do catalisador, conforme observado nas Figuras 7 e 8, em que se nota o rápido decréscimo da concentração, apresentada na forma de uma variável adimensional. Essa concentração chega a ser nula no interior do catalisador para reações exotérmicas, como conseqüência das altas taxas de reação observadas, conforme se verifica

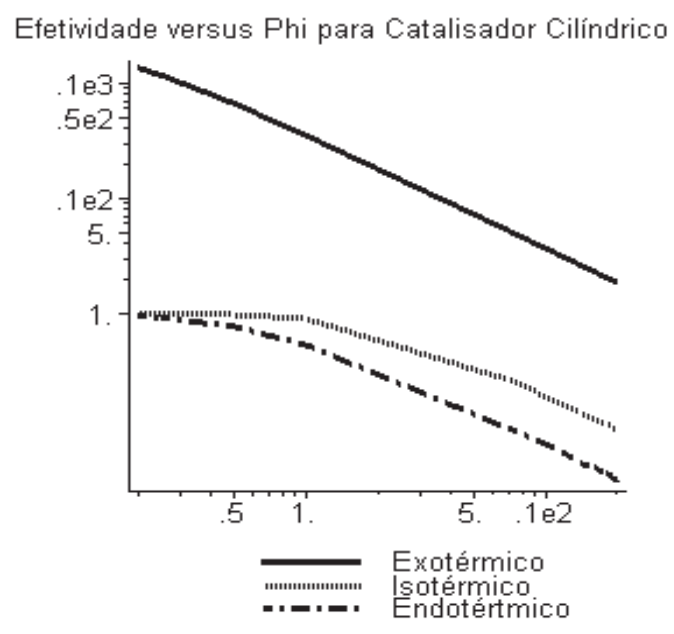

Figura 6. Fator de Efetividade $(\eta)$ em função do Módulo de Thiele $\phi$ (Phi) para catalisador cilíndrico.

Para $\beta=-0,3$ e $\gamma=20$, a Figura 7 indica que conforme os valores de Módulo de Thiele $(\phi)$ aumentam de 0,5 a 4 , os gradientes de concentração e temperatura ao longo do grão catalítico se tornam maiores. Verifica-se que para $\phi=4$, a concentração converge para um valor próximo de 0,65 , a $x=r / R$ $=0,2$, enquanto a temperatura converge a um valor próximo de 0,8 . Já para $\phi$ igual a 0,5 praticamente não se observa variação de concentração nem de temperatura. Como o Módulo de Thiele está relacionado ao tamanho do grão, à medida que o diâmetro do grão aumenta maiores são os problemas de difusão interna de reagentes e produtos. Além disso, há que se considerar que as reações endotérmicas, quando sujeitas às limitaçóes difusionais de calor e massa, provocam o decréscimo da temperatura no interior do grão, pois o calor não é suprido imediatamente após cada ciclo reacional. Conseqüentemente, este decréscimo de temperatura provoca uma queda nas velocidades da reação.
Já para as reações exotérmicas, a temperatura no interior do grão catalítico aumenta conforme a reação se processa, como conseqüência da dificuldade de se transferir o calor gerado pela reação para o meio, devido à limitação à transferência de calor. Pela Figura 8, pode-se verificar os efeitos que as variações no valor de $\beta$ provocam nos perfis de concentração e temperatura. Valores crescentes de $\beta$, que significam reações mais exotérmicas, provocam maiores limitações difusionais sendo que para $\beta=0,3$ a reação fica praticamente restrita à superfície externa. Conforme a temperatura no interior do grão aumenta, a velocidade de reação torna-se mais rápida, consumido rapidamente o pouco reagente que conseguiu penetrar no grão. Isto faz com que os valores de concentração tornem-se nulos à distância próxima da superfície.

Considerando que os resultados normalmente apresentados na literatura são geralmente nas formas de gráficos de $\eta$ em função de $\phi$, para os valores inteiros de $\gamma$ iguais a 10, 20, 30 e 40, a metodologia aqui apresentada possibilita variações intermediárias nos valores dos parâmetros, conforme os observados industrialmente, permitindo um estudo mais amplo.

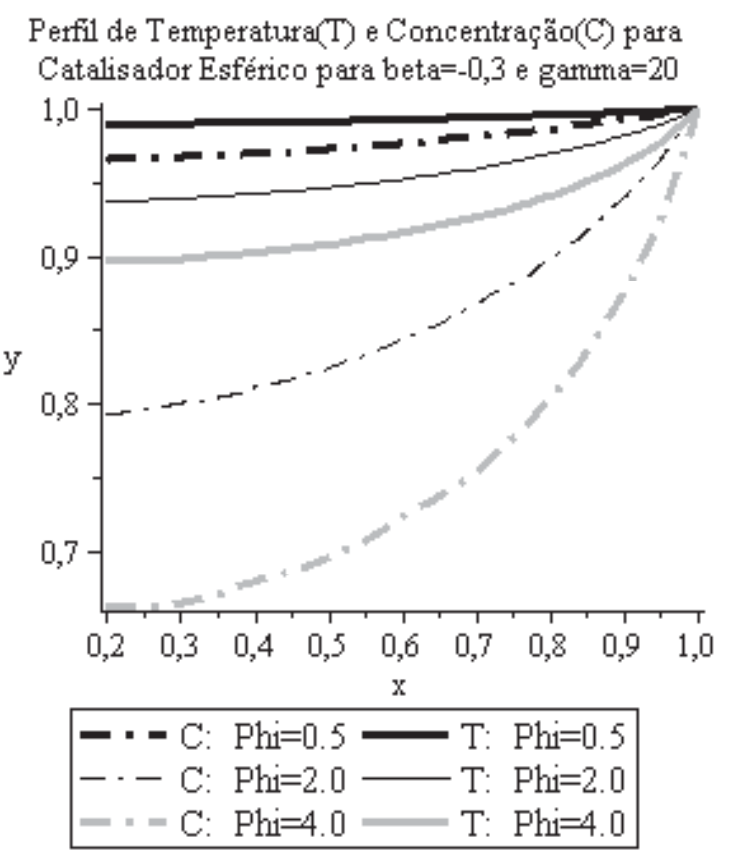

Figura 7. Perfil de Temperatura e Concentração para Reaçóes Endotérmicas $(\beta=-0,3$ e $\gamma=20)$ 


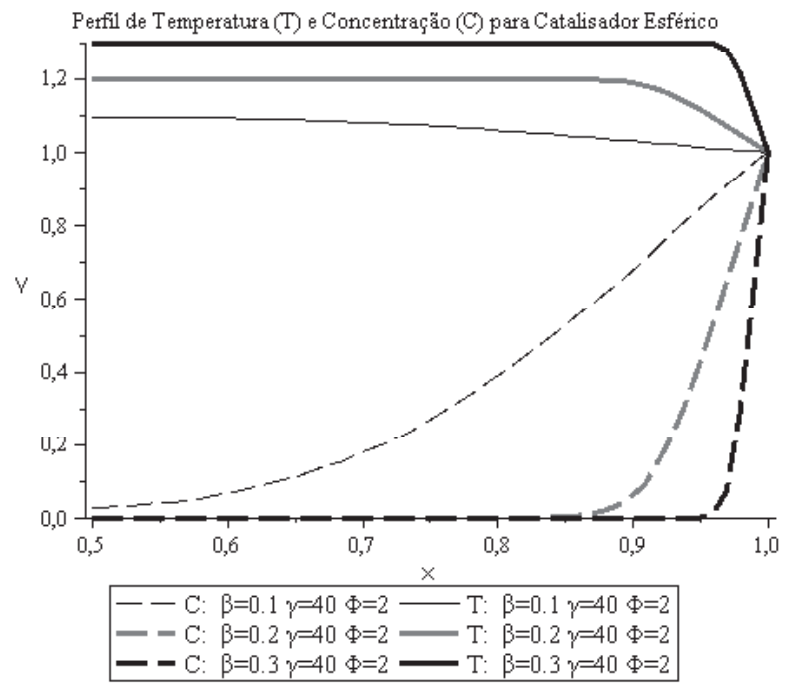

Figura 8. Perfil de Temperatura e Concentração para Catalisadores

Esféricos em Reaçóes Exotérmicas, para vários valores de $\beta$.

\section{CONSIDERAÇÔES FINAIS}

O uso do ambiente computacional usando o software MAPLE é interessante para solucionar problemas complicados como esse apresentado, tornando o ensino mais facilitado.

No presente trabalho, utilizou-se o MAPLE para modelar o sistema de equações diferenciais resultantes de reações químicas catalíticas sujeitas às limitações difusionais internas à transferência de calor e massa, apresentando uma abordagem seqüencial.

Os resultados são apresentados na forma de gráficos em função dos parâmetros $\phi, \gamma$ e $\beta$, permitindo-se avaliar os valores do fator de efetividade, $\eta$, para os valores de módulos de Thiele, $\phi$, propostos.

A visualização dessas soluções de forma interativa faz com que o usuário perceba as influências dos diversos fatores, pois ele pode escolher os parâmetros, verificar suas influências e decidir os novos caminhos. O problema aqui proposto pode ter uma série de variações, que podem ser analisadas. Por exemplo, a forma do catalisador como visto, a ordem da reação, os valores de $\beta$ que podem variar de valores negativos a positivos, geralmente na prática variam de $-0,5$ até 0,5 , passando pelo zero (reaçōes isotérmicas). Os valores de $\gamma$ podem assumir qualquer valor positivo, comumente estão entre 0 e 60, e os valores de módulo de Thiele, também positivos, podem assumir valores acima de zero e à medida que crescem, como já foi dito, aumentam as limitaçōes difusionais internas.

Isto visto, a utilização deste software propicia um entendimento maior do assunto. É uma ferramenta que permite fazer os cálculos em qualquer momento, para qualquer hipótese, sem ter que recorrer a gráficos ou tabelas, pré-existentes e limitados.

Didaticamente, representa uma alternativa promissora, um avanço na forma de ensinar. A utilização desta ferramenta torna o aprendizado mais dinâmico e voltado à análise conceitual, fazendo com que o usuário, com base nos resultados que vão sendo obtidos, venha a decidir os novos parâmetros a serem inseridos, já que o software permite uma abordagem seqüencial.

\section{NOMENCLATURA:}

$C_{A}$ Concentração molar do componente $\mathrm{A}(\mathrm{mol} /$ $\mathrm{cm}^{3}$ )

$D_{\text {Aef }}$ Difusividade Efetiva

$\Delta \mathrm{H}_{R}$ Entalpia da reação

$r$ Raio do grão

$-r_{A}$ Taxa de reação (taxa de desaparecimento do componente A)

$T$ Temperatura (K)

$T_{S}$ Temperatura na superfície do grão $(\mathrm{K})$

$x$ Parâmetro adimensional que representa a distância adimensional ao longo do grão catalítico.

y Parâmetro adimensional que pode representar concentração ou temperatura.

\section{LETRAS GREGAS:}

$\beta \quad$ Parâmetro adimensional definido por Weiss e Hicks relativo a exotermicidade da reação

$\gamma$ Parâmetro adimensional definido por Weiss e Hicks relativo a energia de ativação da reação.

$\eta \quad$ Fator de efetividade (adimensional)

$\rho \quad$ Densidade do grão catalítico $\left(\mathrm{g}_{\mathrm{cat}} / \mathrm{cm}^{3}\right)$

$\phi \quad$ Módulo de Thiele (adimensional)

$\lambda$ Condutividade témica do grão

\section{REFERÊNCIAS BIBLIOGRÁFICAS}

ABEL, M. L.; et al. (1999). MAPLE V by Example New York: Academic Press. 
CALDAS, F. V., SILVA, R. R. C. M. ROCHA, A. A. (2005a). Ensinando Fenômenos de Transporte em um Ambiente Computacional Interativo Usando o Maple. In: XI Encontro de Educação em Engenharia, Penedo.

CALDAS, F. V., SILVA, R. R. C. M. ROCHA, A. A. (2005b). Modelagem de problemas de reação em catalisadores porosos sujeitos à limitação difusional interna de calor e massa utilizando um ambiente computacional interativo utilizando o MAPLE. In: IV Seminário Fluminense de Engenharia, UFF, Niterói.

CREMASCO, M. A. (2004). Fundamentos de Transferência de Massa, Editora da Unicamp.

FIGUEIREDO, J. I.; RIBEIRO, F. R. (1989). Catálise Heterogênea. Lisboa: Fundação Calouste Gulbenkian.

FOGLER, H. S. (2002). Elementos de Engenharia das Reações Químicas. Ed. Livros Técnicos e Científicos, Rio de Janeiro, RJ. Traduzido por Flávio Faria de Moraes e Luismar Marques Porto, da 3a ed. em inglês (1999) Elements of Chemical Reaction

FROMENT, G.F. BISCHOFF, K.B. (1979). Chemical Reactor Analysis and Design. New York. Wiley.

HECK, A. (1993). Introduction to Maple New York: Springer-Verlag.
INCROPERA, F. P.; et al. (2003). Fundamentos de transferência de calor e de massa: LTC-Livros técnicos científicos editora, Rio de Janeiro.

MAYMO, J. A.; SMITH, J. M. (1966). Catalytic Oxidation of Hydrogen - Intrapellet Heat and Mass Transfer. A.I.Ch.E. Journal, vol. 12, no 5, pp. 845-854.

MEADE, D. B.; HARAN, B. S.; WHITE, R. E. (1996). The Shooting Technique for the Solution of Two-Point Boundary Value Problems. Mapletech Computer Algebra, vol. 3, no 1. South Carolina: Waterloo Maple Inc.

NIJEMEISLAND, M., DIXON, A. G. E STITT, (2004) E. H. Chemical Eng. Science, 59, pp. $5185-5191$.

SATERFIELD, C. N. (1991). Heterogeneous Catalysis in Industrial Practice, $2^{\text {nd }}$ edition. New York. McGraw-Hill.

TWIGG, M. V. Catalyst Handbook, 2nd edition, edited by Martyn V. Twigg, pp. 225.

WEISZ, P. B.; HICKS, J. S. (1962). The Behaviour of Porous Catalyst Particles in View of Internal Mass and Heat Diffusion Effects. Chemical Engineering Science, vol. 17, pp. 265-275. London: Pergamon Press Ltd. 